\title{
Biological significance of sperm whale responses to sonar: comparison with anti-predator responses
}

\author{
Charlotte Curé $^{1, *}$, Saana Isojunno ${ }^{2}$, Fleur Visser ${ }^{3,4}$, Paul J. Wensveen ${ }^{2}$, Lise D. Sivle ${ }^{5}$, \\ Petter H. Kvadsheim ${ }^{6}$, Frans-Peter A. Lam ${ }^{7}$, Patrick J. O. Miller ${ }^{2}$ \\ ${ }^{1}$ CEREMA - DTer Est, Acoustics Group, 67035 Strasbourg Cedex 2, France \\ ${ }^{2}$ Sea Mammal Research Unit, Scottish Oceans Institute, University of St Andrews, St Andrews, Fife KY16 8LB, UK \\ ${ }^{3}$ Kelp Marine Research, 1624 CJ, Hoorn, The Netherlands \\ ${ }^{4}$ Behavioural Biology Group, Leiden University, 2300 RA, Leiden, The Netherlands \\ ${ }^{5}$ Institute of Marine Research, 5005 Bergen, Norway \\ ${ }^{6}$ Norwegian Defence Research Establishment (FFI), 3191 Horten, Norway \\ ${ }^{7}$ Netherlands Organization for Applied Scientific Research (TNO), 2597 AK, The Hague, The Netherlands
}

\begin{abstract}
A key issue when investigating effects of anthropogenic noise on cetacean behavior is to identify the biological significance of the responses. Predator presence can be considered a natural high-level disturbance stimulus to which prey animals have evolved adaptive response strategies to reduce their risk of predation by altering behavior away from fitness-enhancing activities such as foraging. By contrasting the type and magnitude (duration, severity, consistency) of behavioral responses to anthropogenic noise and playback of killer whale (KW) sounds that simulated predator presence, this study aimed to provide a relative index of the disturbance level as an indication of the biological significance of responses to the anthropogenic stimulus. Using multi-sensor tags as well as visual observations of surface behavior of adult male sperm whales, we assessed a comprehensive range of behavioral metrics that could reduce individuals' fitness if altered for a biologically relevant duration. Combining previously published results and new analyses, we showed that the responses to $1-2 \mathrm{kHz}$ upsweep naval sonar and to KW playback were very similar, including horizontal avoidance, interruption of foraging or resting activities and an increase in social sound production. However, only KW playbacks elicited grouping behaviors, indicating that this social response component was specific to predator detection. Animals responded to a lesser extent to $6-7 \mathrm{kHz}$ upsweep naval sonar, indicating weaker disturbance effects. Our study demonstrates the benefit of using anti-predator responses as a reference of disturbance when evaluating the relative impacts of anthropogenic stimuli, which can be of particular interest in studies of threatened species such as sperm whales.
\end{abstract}

KEY WORDS: Sperm whales - Behavioral responses - Naval sonar - Anti-predator responses · Anthropogenic disturbance

\section{INTRODUCTION}

Marine mammals, and particularly cetacean species, rely on acoustics as a primary sensory modality to communicate, reproduce, find prey, orientate and obtain information from their environment (e.g. detection of predators) (Richardson et al. 1995, Tyack

\footnotetext{
${ }^{*}$ Corresponding author: charlotte.cure@cerema.fr
}

2008). Underwater anthropogenic noise can impact cetaceans in different ways, including physiological effects (e.g. increasing level of stress or hearing impairment) and behavioral changes (e.g. avoidance responses) (Nowacek et al. 2007, Southall et al. 2007). Physiological effects are commonly investigated under laboratory conditions for those species that can be

() The authors 2016. Open Access under Creative Commons by Attribution Licence. Use, distribution and reproduction are unrestricted. Authors and original publication must be credited. 
maintained in captivity, whereas behavioral effects are generally more context-dependent and can only be fully evaluated on free-ranging animals.

Our ability to understand and manage the effects of anthropogenic noise on free-ranging cetacean behavior is impeded by the difficulty in identifying the level of potential disturbance and interpreting its biological significance. The biological importance of anthropogenic impacts should be investigated by examining long-term effects on critical biological population metrics such as growth, survival, reproduction and distribution (Wartzok et al. 2003, Southall et al. 2007). However, these metrics require a large and representative sample from the target population, which is often difficult to obtain and can lead to uncertainty in the results. Therefore, most behavioral response studies have concentrated on establishing links between short-term behavioral changes that can have a possible relevance to individual fitness and long-term population consequences (New et al. 2014).

In order to improve our ability to assess behavioral disturbance effects of a given anthropogenic noise stimulus, an alternative approach is to compare responses to this stimulus with responses to a natural and well-known high-level threatening stimulus (such as predator stimuli presentations) as a reference model of disturbance. Such a reference model should provide a template of how animals have evolved to react when faced with a natural biological disturbance (Frid \& Dill 2002). Predator presence represents the highest level of acute threat that animals can face in natural conditions, as it represents a risk of lethal attack from the predator; thus, costly behavioral responses are expected to evolve if they effectively reduce predation risk (Lima \& Dill 1990). Anti-predator behaviors can be transmitted genetically, or learned from individual experiences through direct interactions with predators and/or through observations of conspecifics or heterospecifics interacting with predators (social learning) (Ferrari \& Chivers 2008). Particularly in cases of strong natural selection in the past, anti-predator responses can be expected to be clear and consistent among individuals within species (relative to more evolutionarily novel stimuli; Sih 2013), providing a clear and reliable template of high-level disturbance. An animal's decision to respond to a threat (such as predator presence) is based upon a trade-off between the costs and benefits of behavioral change and the perceived risks evaluated by the animal (Frid \& Dill 2002, Brown \& Kotler 2004, Sih 2013).
Thus, animals must make a choice between avoiding the perceived risk from the threat versus continuing fitness-enhancing activities (e.g. feeding or maternal care), particularly if the activities themselves increase predation risk. Analyses of behavioral changes of various animal species in the presence of predators and anthropogenic stimuli suggest that animals have evolved anti-predator responses that shape their responses to human disturbance (Frid \& Dill 2002). Therefore, predation risk and anthropogenic disturbance stimuli can generate similar trade-offs. Assessing the degree of similarity between the behavioral responses to predator presence (the reference model) and to anthropogenic stimuli could therefore be a valuable approach to indicate the relative level of disturbance caused by anthropogenic stimuli in a given decision-making context.

Among the various underwater anthropogenic noise sources impacting cetaceans, naval long-range active sonars are of particular concern since their use has been linked to several cetacean mass stranding events (Frantzis 1998, Balcomb \& Claridge 2001, D'Amico et al. 2009). These sonar signals are generated at high power levels (rms source levels between 216 and $240 \mathrm{~dB}$ re $1 \mu \mathrm{Pa}$ at $1 \mathrm{~m}$ ) within the 0.1 to $10 \mathrm{kHz}$ frequency band (Hildebrand 2009, Ainslie 2010), which is within the hearing range of most cetacean species (Popper \& Ketten 2008). Necropsies of stranded animals have given indications of physical injuries caused directly or indirectly by naval sonar (Evans \& England 2001, Jepson et al. 2003, Fernández et al. 2005, Cox et al. 2006). In addition, a number of behavioral response studies have demonstrated that sonar can also affect cetacean behavior at the individual and group level in a manner judged to be severe (e.g. cessation of feeding, separation from offspring) (Tyack et al. 2011, Miller et al. 2012, 2015, DeRuiter et al. 2013, Goldbogen et al. 2013, Sivle et al. 2015, Isojunno et al. 2016), indicating a potential for fitness consequences if sonar exposures were sufficiently common and animals continued to respond. Field research with free-ranging cetaceans is costly, requires intense logistics and usually obtains a low number of samples. Moreover, behavioral responses to sonar have been found to be highly variable not only across species but also among individuals within species (Miller et al. 2012, Sivle et al. 2015), increasing the difficulty in identifying behavioral responses that may indicate a disturbance state, and to assess the biological relevance and significance of those responses.

In the present work, we investigated the degree of similarity between the behavioral responses of adult 
male sperm whales Physeter macrocephalus in northern Norway to predator presence (reference model) and to naval sonar by evaluating both the type and magnitude of the responses (duration, severity, and consistency across individuals). If the animals employ similar behavioral strategies when facing 2 different types of disturbances, we can expect that they perceive the 2 stimuli as being a similar level of risk. Understanding the underlying mechanisms driving behavioral responses will enable us to make better predictions for species and anthropogenic disturbance types that have not been studied directly. In order to induce anti-predator responses, we wanted to expose animals to a predator stimulus that might simulate predator presence as naturally as possible. To do so, we chose to simulate predator presence acoustically, by playing killer whale sounds (Curé et al. 2013).

The sperm whale has been classified as Vulnerable in the IUCN Red List of Threatened Species. After almost a century of large-scale commercial whaling that continued until the 1980s, the sperm whale stock underwent a global population decline, which is currently slowly recovering from depletion. In some areas though, subpopulations are not recovering and even continue to decline, likely due to bycatch in driftnets and chemical and noise pollution factors (Carroll et al. 2014). Previous work has shown that the behavior of sperm whales can be altered in response to naval sonar exposure (Miller et al. 2012, Sivle et al. 2012, Isojunno et al. 2016). Thus, investigations of the possible impact of these behavioral changes in response to sonar need to be conducted.

In order to assess the level of behavioral disturbance from naval sonar on sperm whales and to interpret the biological relevance of their responses, we systematically compared their responses to sonar with their anti-predator responses. Sperm whales can be preyed upon by killer whales Orcinus orca, and exhibit stereotyped anti-predator behaviors in response to the playback of killer whale sounds (Curé et al. 2013). On their feeding grounds, sperm whales typically spend $80 \%$ of their time foraging and $20 \%$ of their time resting and engaging in other activities (Miller et al. 2004, 2008, Sivle et al. 2012). They typically perform long, deep foraging dives (200 to $1500 \mathrm{~m}, 25$ to $50 \mathrm{~min}$ ) separated by 5 to $15 \mathrm{~min}$ surfacing intervals (Sivle et al. 2012). During foraging dives, they produce loud echolocation clicks (indicating prey searching) and buzzes (indicating prey capture attempts) (Miller et al. 2004). While resting, they exhibit stereotypical shallow and short dives (100 m, 20 to $30 \mathrm{~min}$ duration), with no foraging sounds (Miller et al. 2008). Adult male sperm whales are usually solitary, and grouping behavior and/or vocal production of codas (associated with social or alarm function) is rarely observed (Madsen et al. 2002, Lettevall et al. 2002, Whitehead 2006, Frantzis \& Alexiadou 2008, Teloni et al. 2008, Curé et al. 2013). In contrast, adult males commonly produce slow clicks that have been suggested to be associated with communication amongst males on their highlatitude feeding grounds (Oliveira et al. 2013).

To investigate how sperm whale behavioral responses to naval sonar compare to the disturbance effect induced by the detection of a predator (i.e. a killer whale), we focused on 3 primary behavioral aspects: horizontal avoidance responses, alteration of foraging and resting behaviors, and induction of social responses. For each behavioral aspect, we combined previously published results (Miller et al. 2012, Sivle et al. 2012, Curé et al. 2013, Isojunno et al. 2016) with additional data. We assessed a comprehensive range of behavioral response metrics, e.g. changes in diving behavior, reduction in the production of sounds associated with foraging (clicks and buzzes), changes in the direction and speed of horizontal movement, association with other whales, and changes in the production of social sounds (codas and slow clicks). If altered for a biologically relevant duration, such responses have the potential to reduce the fitness of exposed individuals and thus ultimately have consequences at the population level. Therefore, depending upon the risk whales associate with different natural and anthropogenic stimuli, we expect that there should be a gradient in the magnitude of responses in these behavioral parameters. Using the threatened sperm whale as a model species, this work aims to provide such a relative index of behavioral disturbance (type and magnitude) to naval sonar compared to a known high-level behavioral disturbance template (anti-predator responses), and thereby enable indications of the biological significance of the responses.

\section{MATERIALS AND METHODS}

\section{Ethical statement}

All experiments comply with the current laws of the country where they were performed (Norway). Animal experiments were carried out with permission from the Norwegian Animal Research Authority (Permit No. S-2007/61201). Protocols were approved by the Animal Welfare and Ethics Committee of the 
University of St Andrews, UK, and the Institutional Animal Care and Use Committee of the Woods Hole Oceanographic Institute, USA.

\section{General protocol}

The experiments were designed and conducted by the 3S (Sea mammals, Sonar, Safety) research project. The protocol is fully described in Miller et al. (2011, 2012), and in Curé et al. $(2012,2013)$, and only briefly summarized here. Fieldwork was conducted in the northern Norwegian Sea during May and June 2008, 2009 and 2010. The protocol comprised the following phases: (1) tagging operation, where a non-invasive digital acoustic recording tag (DTAG) was attached to the whale by suction cups; (2) baseline data collection of the tagged animal; (3) exposure experiment sessions (sonar, killer whale sound playbacks, controls); and (4) recovery of the tag once it detached from the animal. The DTAG was typically programmed to release from the whale after 15 to $18 \mathrm{~h}$.

Sonar exposures were conducted from a dedicated source vessel (the $55 \mathrm{~m} \mathrm{R} / \mathrm{V}$ 'H. U. Sverdrup II'). Visual tracking of the tagged whale and observations of other nearby whales were conducted from a separate dedicated observation vessel. Tagging operations and sound playbacks were conducted from an additional motor boat launched from one of the 2 research vessels (see detailed protocol in Miller et al. 2011).

\section{Sound exposure experiments}

\section{Controlled exposure experiments (CEE)}

Three types of sonar exposure sessions were conducted from the source vessel. Low frequency active sonar (LFAS) was composed of a hyperbolic upsweep signal with a frequency band from 1 to $2 \mathrm{kHz}$ and maximum source level of $214 \mathrm{~dB}$ re $1 \mu \mathrm{Pa} \mathrm{m}_{i}$ LFASDS was similar but with a downsweep signal (from 2 to $1 \mathrm{kHz}$ ). Mid frequency active sonar (MFAS) was composed of a hyperbolic upsweep signal from 6 to $7 \mathrm{kHz}$ with a maximum source level of $199 \mathrm{~dB}$ re $1 \mu \mathrm{Pa}$ m. Sonar exposure sessions $(\mathrm{n}=10)$ were composed of a $1 \mathrm{~s}$ signal, repeated every $20 \mathrm{~s}$ for a total duration (mean $\pm \mathrm{SD}$ ) of $50 \pm 18$ min (range: 33 to $95 \mathrm{~min}$ Table 1). The sonar source (SOCRATES; Netherlands Organisation for Applied Scientific Research [TNO], The Hague, The Netherlands) was towed by the source vessel towards the focal whale at a depth ranging from 35 to $100 \mathrm{~m}$. The source levels were increased from $152 \mathrm{~dB}$ re $1 \mu \mathrm{Pa}$ m (LFAS / LFAS-DS) or $158 \mathrm{~dB}$ re $1 \mu \mathrm{Pa} \mathrm{m}$ (MFAS) to the maximum source level over the first $10 \mathrm{~min}$ of transmissions. To control for a potential effect of the approaching vessel itself, we conducted a similar approach of the vessel but with no transmission (no-sonar control [CTRL]; $\mathrm{n}=2$ ) (duration: $50 \pm 21 \mathrm{~min}$ ). Received sound pressure levels of sonar exposures ranged from 79 to $169 \mathrm{~dB}$ re $1 \mu \mathrm{Pa}$ (See Miller et al. 2011 for protocol details). The sonar exposures LFAS and MFAS and the no-sonar CTRL were transmitted in an alternated order across experiments and had a recovery period of at least $1 \mathrm{~h}$ between them. LFAS-DS was always transmitted last, after the killer whale playback experiments.

\section{Sound playback experiments}

Sperm whales were exposed to the playback of unfamiliar mammal-feeding killer whale sounds (KW) after having been previously exposed either to naval sonar (at least $2 \mathrm{~h}$ before) or to the playback of a broadband noise (noise CTRL) (30 min before) (Table 1). Both the KW and noise CTRL stimuli lasted approximately $15 \mathrm{~min}$ (range: 15 to $21 \mathrm{~min}$; Table 1) and were broadcast at a frequency band of 0.5 to

Table 1. Tested sperm whales Physeter macrocephalus and sound exposures. Each tagged whale was subjected to a set of sound exposure sessions (duration of exposure in min; order of exposures marked as \#number). LFAS: low frequency active sonar (1 to $2 \mathrm{kHz}$ ); LFAS-DS: downsweep LFAS; MFAS: mid-frequency active sonar (6 to $7 \mathrm{kHz}$ ); no-sonar CTRL: sonar exposure protocol but with no sonar transmission; KW: killerwhale sound playback; noise CTRL: broadband noise playback

\begin{tabular}{|c|c|c|c|c|c|c|}
\hline ID of exposed animals & LFAS & LFAS-DS & MFAS & No-sonar CTRL & KW & Noise CTRL \\
\hline Sw08_152a & $61 \min _{i} \# 2$ & & 95 min; \#1 & & & \\
\hline Sw09_141a & $40 \mathrm{~min}$; \#1 & & $36 \mathrm{~min} ; \# 2$ & $64 \mathrm{~min} ; \# 3$ & 21 min;\#4 & \\
\hline Sw09_142a & $44 \min _{i} \# 2$ & $50 \min _{i} \# 5$ & $33 \min _{i} \# 3$ & $35 \min _{i} \# 1$ & $19 \min _{i} \# 4$ & \\
\hline Sw09_160a & $43 \min _{;} \# 2$ & $59 \min _{i} \# 4$ & $42 \min ; \# 1$ & & $19 \min _{;} \# 3$ & \\
\hline Sw10_149a & & & & & $15 \min _{i} \# 2$ & $15 \min _{i} \# 1$ \\
\hline Sw10_150a & & & & & $15 \min _{i} \# 2$ & $15 \min _{;} \# 1$ \\
\hline
\end{tabular}


$20 \mathrm{kHz}$ (most energy within 1 to $10 \mathrm{kHz}$ ). KW sounds were previously recorded in the North Pacific using DTAGs (Curé et al. 2013). The KW playback stimulus was used as a biologically-relevant reference (positive control of disturbance) to contrast with responses to sonar. The noise CTRL stimuli corresponded to amplified non-calling periods of the recordings from which KW stimuli were taken, and served as a negative control of the KW playbacks to assess whether or not anti-predator responses were specific to KW sounds and not to any other sounds played by the speaker. The playback sounds were generated by a M-Audio Microtrack II recorder amplified by a Cadence Z8000 amplifier connected to a Lubell LL4642T loudspeaker (frequency response: 0.2 to $20 \mathrm{kHz}$ ) deployed underwater at a depth of $8 \mathrm{~m}$. Each playback experiment was conducted from a dedicated small motor boat positioned roughly $800 \mathrm{~m}$ from the focal whale (boat drifting, with engine off during playback). KW and CTRL playback source levels were lower than the sonar source levels. The average rms source level of both playbacks stimuli (KW and noise CTRL) ranged from 145 to $151 \mathrm{~dB}$ re $1 \mu \mathrm{Pa} \mathrm{m}$ (Curé et al. 2013) which corresponds to the average sound level of KW vocalizations observed under natural conditions (Miller 2006). Estimated average received levels of KW and CTRL stimuli at the start of playback (Curé et al. 2012) ranged between 94 and $105 \mathrm{~dB}$ re $1 \mu \mathrm{Pa}(\mathrm{n}=7$ playback sessions). To avoid pseudoreplication, 3 different versions of stimuli (coming from 3 different recordings) were used for each stimulus type (KW and CTRL) (McGregor et al. 1992)

\section{Analysis of the behavioral responses}

To contrast the behavioral changes associated with naval sonar transmissions versus those from predator sound playbacks, we focused on 3 primary sperm whale behavioral aspects: horizontal movement (heading and speed), foraging and resting behaviors (production of foraging sounds and dive pattern), and social responses (grouping behavior and production of social sounds). The analysis of each of these 3 behavioral response types was conducted by integrating a range of different behavioral metrics and by combining previously published results with new analyses using different analytical approaches (Table 2).

\section{Behavioral response metrics}

We monitored the whales' behavioral responses using a high resolution DTAG (Johnson \& Tyack 2003) and detailed visual observations of the tagged individual and other nearby whales at the sea surface. Visual data collection included the horizontal tracking of the focal whale and surface behavioral sampling (Visser et al. 2014).

\section{Vocal production}

Sound files recorded on the DTAGs were viewed as spectrograms using Adobe Audition software (Blackman-Harris window, FFT length: 4096, time

Table 2. Analysis approaches and literature sources used for combining results on each type of sperm whale Physeter macrocephalus behavioral response parameter. All analyses used the dataset presented in Table 1; Tables S1-S5 are in the Supplement at www.int-res.com/articles/suppl/n031p089_supp.pdf

\begin{tabular}{|c|c|c|}
\hline Behavioral response parameter & Analysis approach & Sources \\
\hline Horizontal movement (Table S3) & $\begin{array}{l}\text { Qualitative severity scoring method } \\
\text { Descriptive analysis }\end{array}$ & $\begin{array}{l}\text { Miller et al. (2012), new data (Table S1) } \\
\text { Curé et al. (2013) }\end{array}$ \\
\hline $\begin{array}{l}\text { Dive profile (vertical movement) } \\
\text { (Table S4) }\end{array}$ & $\begin{array}{l}\text { Qualitative severity scoring method } \\
\text { Univariate analyses of max depth and } \\
\text { dive duration } \\
\text { Descriptive analysis }\end{array}$ & $\begin{array}{l}\text { Miller et al. (2012), new data (Table S1) } \\
\text { Curé et al. (2013) } \\
\text { Sivle et al. (2012), Curé et al. (2013) }\end{array}$ \\
\hline $\begin{array}{l}\text { Foraging/resting activities } \\
\text { (Table S4) }\end{array}$ & $\begin{array}{l}\text { Qualitative severity scoring method } \\
\text { Univariate analyses of \% time of the } \\
\text { dives with production of regular clicks, } \\
\text { and number of buzzes } \\
\text { Hidden state model used for assessing } \\
\text { behavioural state switching }\end{array}$ & $\begin{array}{l}\text { Miller et al. (2012), new data (Table S1) } \\
\text { Curé et al. (2013) } \\
\text { Isojunno \& Miller (2015), } \\
\text { Isojunno et al. (2016) }\end{array}$ \\
\hline $\begin{array}{l}\text { Group distribution } \\
\text { (Table S5) }\end{array}$ & $\begin{array}{l}\text { Qualitative severity scoring method } \\
\text { Descriptive analysis }\end{array}$ & $\begin{array}{l}\text { Miller et al. (2012), new data (Table S1) } \\
\text { Curé et al. (2013) }\end{array}$ \\
\hline $\begin{array}{l}\text { Production rate of codas and } \\
\text { slow clicks (Table S5) }\end{array}$ & $\begin{array}{l}\text { Qualitative severity scoring method } \\
\text { Descriptive analysis }\end{array}$ & $\begin{array}{l}\text { Miller et al. (2012), new analysis (Table S1) } \\
\text { Curé et al. (2013), new data (Table S2) }\end{array}$ \\
\hline
\end{tabular}


resolution: $21.3 \mathrm{~ms}$ ). On the spectrograms, we identified typical vocalizations in the sperm whale vocal repertoire: regular echolocation clicks and buzzes (associated with foraging behavior) (Miller et al. 2004) as well as codas and slow echolocation clicks (associated with social behavior) (Frantzis \& Alexiadou 2008, Oliveira et al. 2013).

Dive behavior

We used the pressure sensor on the DTAG (sampled at $50 \mathrm{~Hz}$ and decimated to $5 \mathrm{~Hz}$ for analysis) to investigate potential changes in dive patterns (vertical movements) in response to the sound exposures. Typical feeding (Miller et al. 2004) and resting dives (Miller et al. 2008) could be clearly identified based on characteristics of duration, depth and acoustic parameters (e.g. presence of buzzes indicating feeding activity).

Direction and speed of horizontal movement

Aided by the VHF radio beacon on the DTAG, we were able to visually track the position of the tagged animal when it came to the sea surface. During each surfacing phase, whale positions were determined at regular 2 min intervals, resulting in 3 to 8 recorded positions per surfacing phase. Potential changes in direction of horizontal movement (avoidance or attraction) were investigated by inspecting the visual tracks of each tagged whale. Horizontal speed was approximated by calculating the ratio between the distance and time between 2 successive sightings of the whale at the surface.

\section{Group composition}

Simultaneously with visual recording of the tagged whale positions at the surface, we scored group size, defined as the number of individuals within $200 \mathrm{~m}$ of the focal animal (Visser et al. 2014).

\section{Analytical approaches}

In this study we integrated new data with the results of previously published papers (Table 2; all of which were derived from the same field studies described above), in order to contrast sperm whale responses to sonar and KW playback. The analyses of the behavioral responses used in these previous publications fall into 4 categories: (1) univariate analyses, (2) state-switching models, (3) expert severity scoring and (4) descriptive analyses.

(1) Univariate analyses were used to assess changes in dive duration, maximum dive depth and production of regular clicks and buzzes in response to KW sound playbacks (Curé et al. 2013). For each of these behavioral parameters, a Wilcoxon matched-pair test (suitable for small sample size) was applied to assess difference between baseline and KW playback.

(2) A hidden state-switching model was developed to estimate (in a Bayesian framework) time series of 6 functional behavioral states from multiple streams of input data including depth, pitch and presence/ absence of regular echolocation clicks (Isojunno \& Miller 2015). The 6 behavioral states included different phases of the foraging activity and additional non-foraging states: animal at the surface, in the descent phase of a foraging dive, in a layer-restricted food searching phase (typically at the bottom of a foraging dive), in the ascent phase of the foraging dive, drifting rest, and in a non-foraging active state. The estimated time series of behavioral states was used to test whether sperm whales switched from foraging or resting states to other behavioral states in response to all exposure types (Isojunno et al. 2016).

(3) An expert scoring of putative responses was used to evaluate the severity of identified behavioral responses on a numeric scale (Southall et al. 2007) ranging from no effect (0), effects not likely to influence vital rates (scores 1 to 3), effects that could affect vital rates (scores 4 to 6), to effects that are likely to affect vital rates (scores 7 to 9 ). The severity score of a response depends on the nature of the response and its duration relative to the duration of the exposure (Southall et al. 2007, Miller et. al. 2012, Sivle et al. 2015). The behavioral responses in the sperm whale dataset of 2008-2009 to sonar signals, no-sonar CTRL, KW sounds and noise CTRL were scored by Miller et al. (2012); the 2010 dataset which included playbacks of KW sounds and noise CTRL were scored in the present work in the exact same way (new data, Table S1 in the Supplement at www.int-res.com/articles/suppl/ n031p089_supp.pdf). The experimental exposures were described and scored by 2 independent scorer groups in accordance with the severity scale. One group consisted of authors C.C., S.I., P.W. and P.M., and the second of authors F.V., L.S., P.K. and F.P.L. Both groups conducted a separate scoring, blind to each other's scoring. Thereafter, the 2 groups met and assimilated their results in the presence of an adjudicator to reach a consensus scoring. Six of the 8 scorers 
had participated in the previous scores presented in Miller et al. (2012); the adjudicator was also the same as in Miller et al. (2012), therefore, we were confident that the new scoring effort (in the present study) followed the same method as in Miller et al. (2012). The severity scores of the following 8 behavioral metrics were systematically recorded for each exposure experiment: avoidance (vertical and horizontal), change in orientation other than avoidance, change in locomotion (speed and directivity), change in dive profile, cessation of foraging (based on alteration of the production of regular clicks and buzzes), cessation of resting, cessation/modification of vocal behavior (including production of social sounds) and change in group distribution (group size).

(4) Descriptive analyses were used to assess changes in the dive parameters (dive duration and maximum dive depth) in response to naval sonar, no-sonar CTRL and noise CTRL playbacks, by comparing data during each exposure session to the exposed animal's own baseline (Sivle et al. 2012, Curé et al. 2013). A descriptive approach was also previously conducted to investigate the changes in direction of horizontal movement and changes in the group composition and coda production in response to KW sounds and noise CTRL playbacks (Curé et al. 2013). In addition, the present study provides a new descriptive analysis assessing occurrences of slow clicks and codas in response to all exposure types (Table 2). In this analysis, the presence or absence of slow clicks and codas within each $1 \mathrm{~min}$ bin in the time series was recorded. Then, for each exposure, we scored respectively the number of $1 \mathrm{~min}$ bins with presence of slow clicks and the number of $1 \mathrm{~min}$ bins with presence of codas within the $15 \mathrm{~min}$ period preceding the start of exposure (Pre period), the duration of the exposure session itself (Dur period) and the $15 \mathrm{~min}$ period following the end of exposure (Post period). Since not all exposures lasted $15 \mathrm{~min}$ (Table 1), we normalized the numbers of $1 \mathrm{~min}$ bin with presence of social sounds found within the duration of an exposure to a $15 \mathrm{~min}$ period of time in order to be able to compare social sound production across the Pre, Dur and Post exposure periods.

Indexing the level of disturbance to sonar and control exposures relative to anti-predator behavior

The type and magnitude of responses were compared between each of the stimuli and the anti-predator response template. For each of the 3 categories of behavioral response, the response magnitude was assessed based upon the following 3 criteria:
(1) Proportion of responding animals - indicating a level of consistency as to whether or not a response occurred (whatever the level of this response) across the tested animals. For a given behavioral response type, a proportion of responding animals higher than $50 \%$ was considered to be relevant. Note that a lower confidence was given for LFAS-DS, no-sonar CTRL and noise CTRL because of a low sample size for those exposure types $(n=2)$.

(2) The severity score given to the response that was judged to have the highest probability of impacting a vital rate - It is possible that a particular behavioral change was not given a severity score (score $0=$ no response) but was considered a response based on another analytical approach (e.g. univariate analysis or descriptive analysis).

(3) Occurrence (binary metric yes/no) of horizontal avoidance response, cessation of feeding, production of codas and grouping behavior - these behaviors likely indicate high level of perceived risk, potentially leading to impact on the fitness of individuals.

\section{RESULTS}

A total of 6 sperm whales were tagged and exposed to at least 2 of the 6 sound stimuli. Four whales were exposed to LFAS and MFAS, 2 to LFAS-DS, and 2 to no-sonar CTRL (Table 1). Three of the 4 whales exposed to sonar signals were also exposed to KW playbacks. Two other whales were only exposed to KW and noise CTRL playbacks.

Overall, each of the 3 categories of behavioral responses were exhibited in response to at least one of the sound exposure types (i.e. LFAS, MFAS, LFASDS, KW playbacks, no-sonar CTRL and noise CTRL playback). All tagged whales showed changes in horizontal movement in response to one or more sound exposures. Those changes were induced in response to 14 (out of 19) exposure sessions (see Table S3 in the Supplement), of which 8 corresponded with an avoidance response. All changes in foraging activities (9 out of 15) corresponded to an alteration or cessation of the production of foraging sounds (i.e. regular clicks and buzzes) and were associated with a change in the dive profile (Table S4 in the Supplement). In no case did an animal start to feed as an apparent response to an exposure. Regarding the social response, 2 of the 6 solitary tagged whales exhibited grouping behavior (Table S5 in the Supplement). Moreover, in 7 and 15 of the 19 exposure sessions, tagged animals changed coda and slow click production rates respectively. 
Based on analyses of the 3 main behavioral response types (Tables S3-S5), we indexed the responses of animals to sonar (and control exposures) relative to the responses to KW playbacks that simulated predator presence (Figs. $1 \& 2$ ).

\section{Behavioral response to killer whale sound playbacks}

Behavioral responses to KW playbacks represented the reference index level of responses to a known high-level natural disturbance. Changes in horizontal movement, alteration of foraging/resting activities, and initiation of social response were all induced in response to KW playbacks and had the highest magnitude compared to responses to other stimuli types (Figs. $1 \& 2$ ). According to the definition of Southall et al. (2007), those responses were scored as being particularly severe, meaning that they are likely to have an impact on vital rate if exposed repeatedly and/or for a long duration. Moreover, responses to KW playback showed high consistency across exposed animals (>50\% of responding animals for each behavioral response category) and had notable indications of a high level of perceived risk (horizontal avoidance, cessation of feeding, coda production, grouping behavior), making the responses to KW playback the highest level of disturbance compared to all exposure types (Fig. 2).

\section{Indexing the level of disturbance to sonar and anti-predator behavior template}

Except for grouping behaviors, the type and magnitude of the behavioral responses were very similar between the KW playbacks and LFAS (Figs. 1 \& 2), indicating a high level of behavioral disturbance induced by LFAS. Both stimuli induced a change in horizontal movement in the majority of exposed animals $(\geq 75 \%)$, and at least half of those responding animals exhibited long horizontal avoidance responses that lasted for the duration of the exposure (severity score 6) or longer (a severity score of 7 was given in response to one $\mathrm{KW}$ playback) (Table S3, Fig. 1). Moreover, all animals tested with LFAS and KW sound playbacks switched to shorter and shallower dives (scores 3 and 4) and/or exhibited unusual dive patterns showing multiple vertical wiggles (i.e. short ascents) during the descent phase of the exposure dives (severity scores 1 and 2) (Table S4). Among the animals exposed to both LFAS and KW playbacks, all that were feeding or resting before the start of LFAS and KW exposures interrupted or considerably reduced their activity during exposure (severity scores 5 and 6) (Table S4, Fig. 1). Modifications of social behavioral parameters were found in at least $75 \%$ of the tested animals for both KW and LFAS exposure types but responses to KW playbacks were judged to have more impact on vital rates (severity scores 4 to 6 in $60 \%$ of the responding animals) than LFAS (one severity score of 4) (Fig. 1,

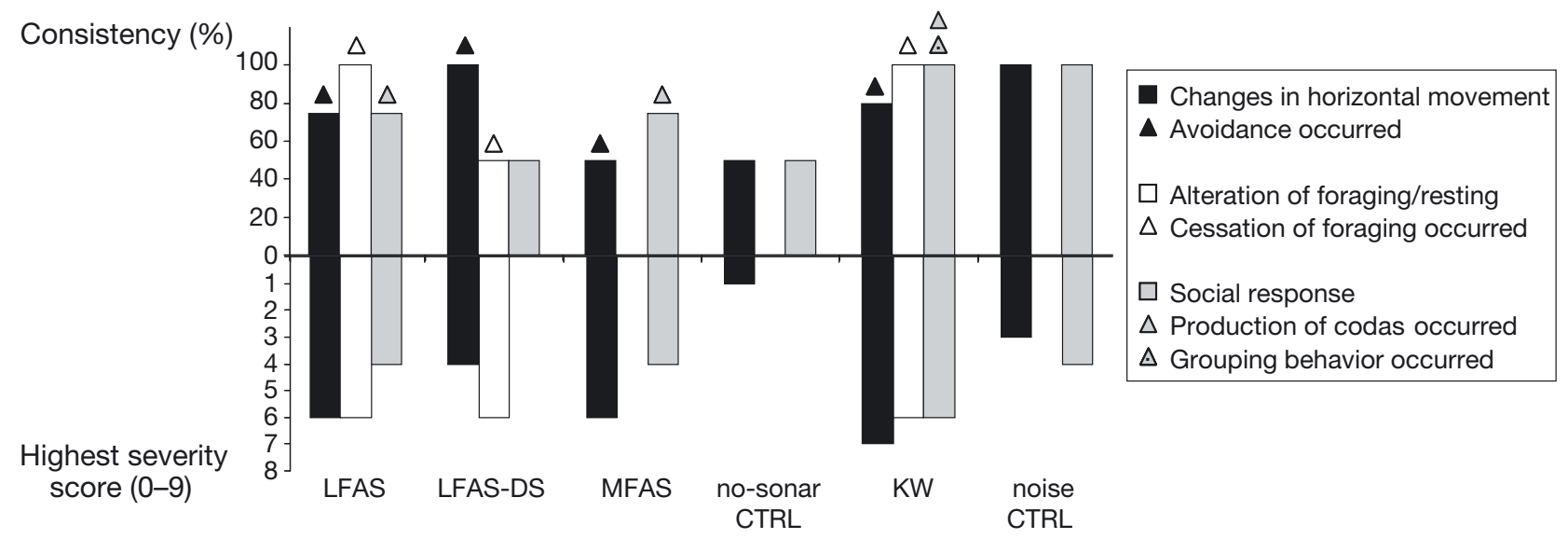

Fig. 1. Type and magnitude of behavioral responses of sperm whales Physeter macrocephalus exposed to different disturbance stimuli, integrating combined results presented in Tables S3-S5 in the Supplement. The magnitude of each of the 3 types of response (changes in horizontal movement, alteration of foraging/resting, and social response) was assessed based on 3 parameters: (1) consistency: the proportion of responding animals, i.e. those showing any changes (whatever the severity of the change), among the range of behavioral metrics describing the corresponding response type (given in Tables S3-S5); (2) highest severity score (scale from 0 to 9): score given to the response judged to have the highest probability of impacting vital rate; and (3) occurrence of particular events (yes/no): avoidance response (for changes in horizontal movement), cessation of feed ing (for alteration of foraging), production of codas and grouping behavior (for social response) 


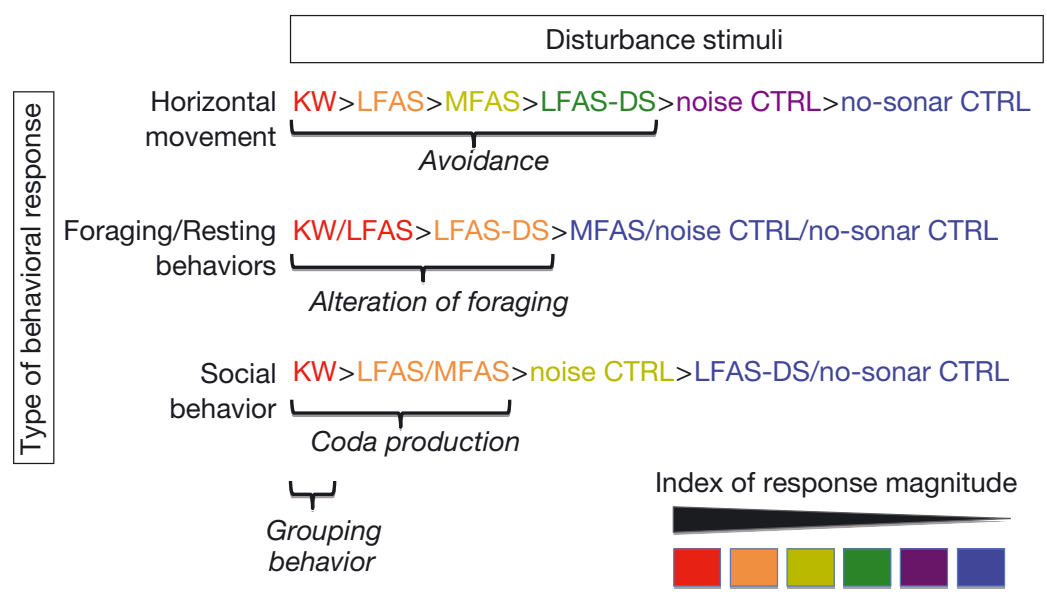

Fig. 2. Schematic representation of the behavioral disturbance index of sperm whales Physeter macrocephalus to different potential disturbance stimuli, including the indexed magnitude of 3 types of responses to killer whale playbacks (i.e. anti-predator responses). KW: killer whale sound playback; LFAS: low frequency active sonar (1 to $2 \mathrm{kHz}$ ); LFAS-DS: downsweep LFAS; MFAS: mid-frequency active sonar ( 6 to $7 \mathrm{kHz}$ ); noise CTRL: broadband noise playback; no-sonar CTRL: sonar exposure protocol but with no sonar transmission

Table S5). Although the grouping response was observed only in $40 \%$ of KW playbacks (group size changed from 1 to $3-4$ whales at the first surfacing following the end of the exposure) and not to LFAS (tagged whales remained solitary), both KW sounds and LFAS exposure types induced a start or increase of coda production and/or an increase of slow click production in at least $75 \%$ of the tested animals, that could last the duration of the exposure (Table S5, Figs. 1 \& 2).

LFAS-DS and MFAS exposures induced only 2 of the 3 behavioral response types, and responses were of lower magnitude compared to responses to KW playbacks and LFAS (Fig. 2). Both of these sonar stimuli induced changes in horizontal movement in at least $50 \%$ of the tested whales, including avoidance responses. However, whereas 2 of 4 MFAS exposures induced a horizontal avoidance response that lasted the duration of the exposure (severity score 6), both LFAS-DS exposures elicited only a brief avoidance response (severity score 4 ) or a minor change in the direction of horizontal movement (not avoidance; severity score 3) (Table S3, Fig. 1). Alteration of foraging behavior (severity score 6) was observed in 1 of the 2 animals exposed to LFAS-DS (sw09_142a) but in none of the 4 whales exposed to MFAS, contrasting with $100 \%$ of foraging animals that stopped or reduced their activity in response to KW and LFAS exposures. Sw09_142a switched from foraging to a non-foraging active and then a resting state during LFAS-DS, whereas it had interrupted resting behavior in response to KW playback and interrupted foraging behavior in response to LFAS. A social response was initiated in response to MFAS but never to LFAS-DS. This social response to MFAS was of similar magnitude as for response to LFAS. In total, $75 \%$ of the animals exposed to MFAS showed increases of codas and slow click production rates, and those changes could last the duration of the exposure (highest severity score: 4).

When presented with control stimuli (i.e. no-sonar CTRL or noise CTRL), animals rarely changed any of the 3 behavioral aspects, and their response magnitude was much lower compared to the KW playbacks and to most of the other exposure types (Fig. 2). Neither horizontal avoidance responses, modifications of foraging activity, nor production of codas were induced in response to the no-sonar CTRL or the noise CTRL. Few changes were found in the direction and/or speed of horizontal movement, with the highest severity scores ranging from 1 for the no-sonar CTRL to 3 for the noise CTRL (Table S5, Fig. 1). Regarding social response, one of the 2 animals exposed to nosonar CTRL and both animals exposed to the noise CTRL increased their slow click production rate during or after the end of exposure. Increased slow click production rate was much higher in response to the noise CTRL exposure than to the no-sonar CTRL since it lasted as long as the duration of the exposure (severity score 4 ).

\section{DISCUSSION}

In this paper, we assessed the behavioral responses of tagged sperm whales to different stimuli: KW sounds simulating predator presence that represented a natural high-level disturbance stimulus, 3 types of naval sonar signals (LFAS, LFAS-DS and MFAS) for which we aimed to fully assess the potential disturbance effect, and 2 control stimuli. The 2 types of control stimuli tested for the specificity of the responses to the sound exposures, in order to ensure that the experimental protocol was not responsible for the observed responses. At least one tagged whale responded to each type of exposure, showing that all stimulus types could be perceived by the sperm whales. Our results demonstrated that overall, 
playback of KW sounds induced the strongest and most consistent behavioral responses, followed by decreasing magnitude of responses to LFAS, LFASDS and MFAS, noise CTRL exposure and then nosonar CTRL, which induced the weakest responses (Fig. 2).

\section{Killer whale playback as a natural high-level disturbance reference model}

The main objective of this study was to identify and understand the disturbance effects of naval sonar by comparing responses to such sonar with anti-predator responses as a reference template of disturbance. As predicted by the potential of being perceived as a high level disturbance stimulus, KW playback generated clear and consistent responses across the 5 exposed animals. KW playbacks induced changes in all 3 behavioral aspects (i.e. alteration of feeding, avoidance responses and initiation of social response) and showed the highest magnitude of response (highest level of consistency across exposed animals, highest probability of impact on vital rates, specific part of response revealing a particularly high level of disturbance) compared to responses to other stimuli. This demonstrates that responses to KW playbacks represent a reliable reference of high-level disturbance that can be used for indexing the level of behavioral disturbance responses (type and magnitude) to other stimuli (Fig. 1).

Responses to KW playbacks gave us indicators used to identify behavioral signs of disturbance in sperm whales: avoidance responses, alteration of foraging and resting, start or increase in coda production and occurrence of grouping behaviors. Start or increase of coda production might reflect a high level of threat and/or the need to coordinate with other whales around in the aim of initiating a social response (Frantzis \& Alexiadou 2008). In contrast, increased slow click production occurred in response to all types of exposure, including some of the controls, suggesting that this particular behavioral change might be a common response to any stimuli, or at least not specifically exhibited in response to the KW sounds or sonar exposures. Based upon the hypothesis that slow clicks function as communication signals (Oliveira et al. 2013), it may serve as signaling the presence of a new stimulus in the environment to other conspecifics in the area.

The behavioral responses to KW playbacks showed that in sperm whales, increases in perceived predation risk can lead to a combination of behavioral changes, including reduced foraging effort and leaving a food patch. If repeated and/or continued, those behavioral changes have potential to impact populations or even species. Moreover, horizontal avoidance in reaction to predators may potentially increase a whale's risk of stranding (Ford \& Reeves 2008, Allen et al. 2014). Such anti-predator responses that reduce individual fitness are expected to evolve if the fitness cost of the response is balanced by a reduction in predation risk (and thereby greater survival).

\section{Contrasting sonar exposure to a natural disturbance model of increasing predation risk}

The behavioral changes in response to KW playbacks indicated the parameters most likely to be altered when sperm whale behavior becomes disturbed by a natural biological stimulus, revealing the most relevant parameters to examine when investigating potential disturbance effects of anthropogenic stimuli on sperm whale behavior. Grouping behavior was induced only in response to KW playbacks, indicating that this part of the social response was specific to the anti-predator context. Responses to LFAS sonar (1 to $2 \mathrm{kHz}$ ) were very similar to responses to KW playbacks, indicating the potential of LFAS to have similar disturbance effects as an increasing predation risk. In contrast, LFAS-DS (downsweep 2 to $1 \mathrm{kHz}$ ) and MFAS sonar signals generally had lower impacts, although they generated a high magnitude response in some behavioral aspects with a disturbance level close to that observed in response to an increased predation risk. Therefore, although tagged animals seemed to be more tolerant to these signals than to LFAS, they still may induce some aspects of the anti-predator response.

Differences between responses to sonar signals versus responses to KW playbacks are clearly not explained by differences in the received level of sound, as the KW playbacks were received at much lower levels than the sonar exposures (maximum levels of 105 vs. $170 \mathrm{~dB}$ re $1 \mu \mathrm{Pa}$ respectively). Since LFAS signals were both transmitted and received at higher sound pressure levels (maximum received SPL $170 \mathrm{~dB}$ re $1 \mu \mathrm{Pa}$ ) than MFAS (maximum received SPL $158 \mathrm{~dB}$ re $1 \mu \mathrm{Pa}$ ) (Isojunno et al. 2016), one could say that stronger responses to LFAS compared to MFAS might be due to higher received sound levels. However, whales started switching from a foraging to an active non-foraging state at a cumulative received sound exposure level (SEL) of 135 to $145 \mathrm{~dB}$ re $1 \mu \mathrm{Pa}$ for 
LFAS, which was $\sim 20 \mathrm{~dB}$ below the maximum cumulative received SEL of MFAS (Isojunno et al. 2016). Therefore, it is unlikely that differences between responses to LFAS and MFAS were due to differences in received sound levels.

One hypothesis to explain the higher level of responses to LFAS compared to other sonar signal types that has already been proposed in Isojunno et al. (2016) is that the lower frequency and higher source level of the LFAS sonar might reflect a bigger threat, in the same way as the dominant frequency of the vocalizations of numerous animal species is known to reflect the size or weight of individuals, with lower values being associated with bigger size (Fletcher 2004, Pfefferle \& Fischer 2006, Bertucci et al. 2012, Mehdipour \& Sendi 2015).

The low sample size of LFAS-DS $(n=2)$ and its potential order effect on the response (given that LFAS-DS was always conducted as the last of 3 sonar exposures), might lead to less reliable conclusions than for the other sonar signals. For the 2 other sonar types (LFAS and MFAS), beside the fact that the order of presentation was well balanced between the 2 (Table 1), no order effect was statistically found regarding the alteration of foraging (Isojunno et al. 2016). Moreover, the present study did not indicate any tendency for a potential order effect on the changes in horizontal movement or social response. For instance, a horizontal avoidance could occur in response to LFAS or MFAS whatever their order of presentation (first or second). Also, although KW playback was never presented first, responses were equally clear in orders ranging from 2 to 4 . Taken together, these observations support that, although based upon a low sample size $(\mathrm{n}=6$ exposed animals), our results are unlikely to be explained by a potential habituation or sensitization (i.e. respectively a progressive reduction or amplification of a response due to repeated exposures). Therefore, other factors must explain the differences in observed behavioral responses to the different anthropogenic stimuli. First, as we observed in our study, responses to a given stimulus can vary among individuals. This inter-individual variability may depend on a range of factors including age, sex, individual experience (e.g. habituation), group size and composition, the individual's body condition, motivational state, range to the stimulus, duration of the exposure and habitat quality (Wartzok et al. 2003). For instance, Tyack (1983) showed that humpback whale groups with calves avoided the playback of conspecific social sounds whereas single animals or groups with no calves displayed an aggressive behavior.
Second, the behavioral context (occurring in feeding or breeding areas) also may lead to different responses. One example is the reactions of bowhead whales to seismic airgun sounds that differed depending on whether the whales were feeding or migrating (Richardson et al. 1986, Miller et al. 2005). Therefore, it is likely that our reference model would need to be adjusted or supplemented with additional experiments in order to take these factors into account and to reliably use that model more broadly in other sperm whale populations.

Overall, the tested animals rarely changed their behavior in response to the control exposures and demonstrated much weaker effects compared to the KW playbacks or to the naval sonar signals. This result indicates that the behavioral response strategies were specifically employed in response to the disturbance stimuli, i.e. detection of predator sounds and sonar signals, and not due to artefacts of protocol or equipment used to conduct the experiments.

Changes in sperm whale behaviors have been observed in response to other anthropogenic noise. For instance, Miller et al. (2009) showed that tagged sperm whales on feeding grounds of the Gulf of Mexico exhibited substantial changes in swimming movements and had reduced buzzing rates, but did not cease foraging or resting activities and did not show avoidance response when exposed to seismic airguns (Miller et al. 2009). Thus, sperm whale responses to airgun sounds did not match the anti-predator behavior template, contrary to the responses to sonar that did in our study (i.e. interruption of feeding, horizontal avoidance). This indicates that airguns were not perceived by sperm whales in the Gulf of Mexico as being the same level of risk as sonar in Norway. Richter et al. (2006) investigated the response of male sperm whales off Kaikoura (New Zealand) to whale watching boats by behavioral observations at the sea surface (no tags were used). They showed that whale watching boats induced an increase in the frequency of changes of horizontal direction of movement, but pointed out their inability to assess biological significance of those responses. Response to KW playback sounds in our study indicated consistent avoidance behaviors rather than erratic movements. Moreover, Richter et al. (2006) showed that non-resident sperm whales in Kaikoura did respond more strongly to whale-watching than the resident whales. Sperm whales in the Gulf of Mexico and Kaikoura are regularly exposed to airgun sounds and whale watching boats, respectively. Therefore, it is plausible that individual animals had sufficient experience and behavioral plasticity to learn more specific behav- 
ioral response strategies for those specific disturbances than a response strategy based upon an antipredator template.

One might conclude that the observed similarities in the responses of cetaceans to naval sonar and KW playbacks indicates that animals are not able to discriminate between the 2, due to similarities in frequency band and modulation of both signal types. This hypothesis appears unlikely given the importance of sound to these animals, which have probably evolved particularly good sound discrimination capacities. However, animals may not always be able to judge the level of risk associated with unfamiliar sounds (such as sonar pings) and might therefore respond as if the sound source constituted a risk similar to that of a predator. Moreover, looking at the overall picture of the behavioral response of the animals to each stimulus type, our results show that sperm whales exhibit different combinations of response type and/or response magnitude across all stimulus types. This indicates that sperm whales are likely able to discriminate among the different sound exposure types. This conclusion is supported by results from Isojunno et al. (2016), who showed different recovery durations in response to LFAS and KW playbacks. Discrimination among the different acoustic stimuli indicates that sperm whales have the capacity to adjust their behavior to the type of disturbance stimulus, revealing evidence of behavioral plasticity in the species. This is important information that might have consequences at the population level, since behavioral plasticity may result in either population vulnerability or resilience depending upon how adaptive the response is to a new disturbance stimulus (Sih 2013).

\section{CONCLUSIONS}

The current study has shown that behavioral responses to playback of predator sounds can be an effective high-level disturbance reference with which to assess the biological significance of behavioral responses to anthropogenic disturbances. Specifically, we have shown that naval sonar may represent a great disturbance to sperm whales, but that this is less severe than a disturbance induced by a naturally occurring increase in predation risk.

Our study has also demonstrated that indexing behavioral responses to anthropogenic stimuli using a combination of quantitative analyses and qualitative expert scoring of putative responses to a known reference model of disturbance provides a useful analytical approach for interpreting the biological significance of the response. This approach is of particular interest for threatened species such as sperm whales, as it allows further development of appropriate management and conservation plans.

Acknowledgements. All research activities were licensed under a permit provided by the Norwegian Animal Research Authority (Permit No. S-2011/38782), and were approved by the Animal Welfare Ethics Committee at the University of St Andrews. The Logger software was made available by the International Fund for Animal Welfare. Research funding was provided by the US Office of Naval Research and the Ministries of Defence of Norway, the Netherlands and France as well as the UK Natural Environmental Research Council. We are grateful to the many researchers and vessel crew members who helped to collect the data, and to Volker Deecke and Ari Shapiro who collaborated with P.J.O.M. to collect the DTAG acoustic recordings of mammal-eating killer whale sounds used in the study.

\section{LITERATURE CITED}

Ainslie MA (2010) Principles of sonar performance modeling. Springer-Verlag, Berlin

Allen AN, Schanze JJ, Solow AR, Tyack PL (2014) Analysis of a Blainville's beaked whale's movement response to playback of killer whale vocalizations. Mar Mamm Sci 30:154-168

Balcomb KC, Claridge DE (2001) A mass stranding of cetaceans caused by naval sonar in the Bahamas. Bahamas J Sci 5:2-12

- Bertucci F, Attia J, Beauchaud M, Mathevon N (2012) Sounds produced by the cichlid fish Metriaclima zebra allow reliable estimation of size and provide information on individual identity. J Fish Biol 80:752-766

Brown JS, Kotler BP (2004) Hazardous duty pay and the foraging cost of predation. Ecol Lett 7:999-1014

Carroll G, Hedley S, Bannister J, Ensor P, Harcourt R (2014) No evidence for recovery in the population of sperm whale bulls off Western Australia, 30 years post-whaling. Endang Species Res 24:33-43

Cox TM, Ragen TJ, Read AJ, Vos E and others (2006) Understanding the impacts of acoustic sound on beaked whales. J Cetacean Res Manag 7:177-187

Curé C, Antunes R, Samarra F, Alves AC, Visser F, Kvadsheim PH, Miller PJO (2012) Pilot whales attracted to killer whale sounds: acoustically-mediated interspecific interactions in cetaceans. PLOS ONE 7:e52201

> Curé C, Antunes R, Alves AC, Visser F, Kvadsheim PH, Miller PJO (2013) Responses of male sperm whales (Physeter macrocephalus) to killer whale sounds: implications for anti-predator strategies. Sci Rep 3:1579

D'Amico AD, Gisiner R, Ketten DR, Hammock JA, Johnson C, Tyack P, Mead J (2009) Beaked whale strandings and naval exercises. Aquat Mamm 35:452-472

DeRuiter SL, Southall BL, Calambokidis J, Zimmer WMX and others (2013) First direct measurements of behavioural responses by Cuvier's beaked whales to midfrequency active sonar. Biol Lett 9:20130223 
Evans DL, England GR (2001) Joint interim report Bahamas marine mammal stranding event of 15-16 March 2000. US Department of Commerce and US Navy, Washington, DC

$>$ Fernández A, Edwards JF, Rodríguez F, Espinosa de los Monteros A and others (2005) "Gas and fat embolic syndrome" involving a mass stranding of beaked whales (Family Ziphiidae) exposed to anthropogenic sonar signals. Vet Pathol 42:446-457

$>$ Ferrari MCO, Chivers DP (2008) Cultural learning of predator recognition in mixed species assemblages of frogs: the effect of tutor-to-observer ratio. Anim Behav 75: 1921-1925

Fletcher NH (2004) A simple frequency-scaling rule for animal communication. J Acoust Soc Am 115:2334-2338

Ford JKB, Reeves RR (2008) Fight or flight: antipredator strategies of baleen whales. Mamm Rev 38:50-86

- Frantzis A (1998) Does acoustic testing strand whales? Nature 392:29

> Frantzis A, Alexiadou P (2008) Male sperm whale (Physeter macrocephalus) coda production and coda-type usage depend on the presence of conspecifics and the behavioural context. Can J Zool 86:62-75

Frid A, Dill LM (2002) Human-caused disturbance stimuli as a form of predation risk. Conserv Ecol 6:11

Goldbogen JA, Southall BL, DeRuiter SL, Calambokidis J and others (2013) Blue whales respond to simulated midfrequency military sonar. Proc R Soc B Biol Sci 280: 20130657

Hildebrand JA (2009) Anthropogenic and natural sources of ambient noise in the ocean. Mar Ecol Prog Ser 395:5-20

Isojunno S, Miller PJO (2015) Sperm whale response to tag boat presence: biologically informed hidden state models quantify lost feeding opportunities. Ecosphere 6:1

Isojunno S, Curé C, Kvadsheim PH, Lam FP, Tyack PL, Wensveen P, Miller PJO (2016) Sperm whales reduce foraging effort during exposure to $1-2 \mathrm{kHz}$ sonar and killer whale sounds. Ecol Appl 26:77-93

Jepson PD, Arbelo M, Deaville R, Patterson IAP and others (2003) Gas-bubble lesions in stranded cetaceans. Nature 425:575-576

Johnson MP, Tyack PLA (2003) Digital acoustic recording tag for measuring the response of wild marine mammals to sound. IEEE J Oceanic Eng 28:3-12

Lettevall E, Richter C, Jaquet N, Slooten E and others (2002) Social structure and residency in aggregations of male sperm whales. Can J Zool 80:1189-1196

Lima SL, Dill LM (1990) Behavioral decisions made under the risk of predation: a review and prospectus. Can J Zool 68:619-640

> Madsen PT, Payne R, Kristiansen NU, Wahlberg M, Kerr I, Møhl B (2002) Sperm whale sound production studied with ultrasound time/depth-recording tags. J Exp Biol 205:1899-1906

McGregor PK, Catchpole CK, Dabelsteen T, Falls JB and others (1992) Design of playback experiments: the Thornbridge Hall NATO ARW consensus. In: McGregor PK (ed) Playback and studies of animal communication. Plenum Press, New York, NY, p 1-9

Mehdipour M, Sendi JJH (2015) External morphology and calling song characteristics in Tibicen plebejus (Hemiptera: Cicadidae). C R Biol 338:103-111

Miller GW, Moulton VD, Davis RA, Holst M, Millman P, MacGillivray A, Hannay D (2005) Monitoring seismic effects on marine mammals — southeastern Beaufort Sea,
2001-2002. In: Armsworthy SL, Cranford PJ, Lee K (eds) Offshore oil and gas environmental effects monitoring: approaches and technologies. Battelle Press, Columbus, $\mathrm{OH}, \mathrm{p}$ 511-542

Miller PJO (2006) Diversity in sound pressure levels and estimated active space of resident killer whale vocalizations. J Comp Physiol A Neuroethol Sens Neural Behav Physiol 192:449-459

Miller PJO, Johnson MP, Tyack PL (2004) Sperm whale behaviour indicates the use of echolocation click buzzes 'creaks' in prey capture. Proc R Soc B Biol Sci 271: 2239-2247

Miller PJO, Aoki K, Rendell LE, Amano M (2008) Stereotypical resting behavior of the sperm whale. Curr Biol 18: R21-R23

Miller PJO, Johnson MP, Madsen PT, Biassoni N, Quero M, Tyack PL (2009) Using at-sea experiments to study the effects of airguns on the foraging behavior of sperm whales in the Gulf of Mexico. Deep-Sea Res I 56:1168-1181

Miller PJO, Antunes R, Alves AC, Wensveen P and others (2011) The 3S experiments: studying the behavioral effects of sonar on killer whales (Orcinus orca), sperm whales (Physeter macrocephalus), and long-finned pilot whales (Globicephala melas) in Norwegian waters. Scottish Oceans Institute, Tech Rep SOI-2011-001. http://soi. st-andrews.ac.uk/documents/424.pdf

Miller PJO, Kvadsheim PH, Lam FPA, Wensveen PJ and others (2012) The severity of behavioral changes observed during experimental exposures of killer (Orcinus orca), long-finned pilot (Globicephala melas), and sperm (Physeter macrocephalus) whales to naval sonar. Aquat Mamm 38:362-401

Miller PJO, Kvadsheim PH, Lam FPA, Tyack PL and others (2015) First indications that northern bottlenose whales are sensitive to behavioural disturbance from anthropogenic noise. R Soc Open Sci 2:140484

New LF, Clark JS, Costa DP, Fleishman E and others (2014) Using short-term measures of behaviour to estimate long-term fitness of southern elephant seals. Mar Ecol Prog Ser 496:99-108

- Nowacek DP, Thorne LH, Johnston DW, Tyack PL (2007) Responses of cetaceans to anthropogenic noise. Mammal Rev 37:81-115

Oliveira C, Wahlberg M, Johnson M, Miller PJ, Madsen PT (2013) The function of male sperm whale slow clicks in a high latitude habitat: Communication, echolocation, or prey debilitation? J Acoust Soc Am 133:3135-3144

Pfefferle D, Fischer J (2006) Sounds and size: identification of acoustic variables that reflect body size in hamadryas baboons, Papio hamadryas. Anim Behav 72:43-51

Popper AN, Ketten DR (2008) Underwater hearing. In: Basbaum A (ed) The senses: a comprehensive reference, Vol 3. Elsevier Press, Oxford, p 225-236

Richardson WJ, Wursig B, Greene CR Jr (1986) Reactions of bowhead whales, Balaena mysticetus, to seismic exploration in the Canadian Beaufort Sea. J Acoust Soc Am 79: 1117-1128

Richardson WJ, Greene CR, Malme CI, Thomson DH (1995) Marine mammals and noise. Academic Press, San Diego, CA

> Richter C, Dawson S, Slooten E (2006) Impacts of commercial whale watching on male sperm whales at Kaikoura, New Zealand. Mar Mamm Sci 22:46-63

Sih A (2013) Understanding variation in behavioural responses to human-induced rapid environmental 
change: a conceptual overview. Anim Behav 85: 1077-1088

Sivle LD, Kvadsheim PH, Fahlman A, Lam FP, Tyack PL, Miller PJO (2012) Changes in dive behavior during naval sonar exposure in killer whales, long-finned pilot whales, and sperm whales. Front Physiol 3:400

Sivle L, Kvadsheim PH, Curé C, Isojunno S and others (2015) Severity of behavioural responses of humpback whale, minke whale and bottlenose whale to naval sonar. Aquat Mamm 41:469-502

Southall BL, Bowles AE, Ellison WT, Finneran JJ and others (2007) Marine mammal noise-exposure criteria: initial scientific recommendations. Aquat Mamm 33:411-522

Teloni V, Mark JP, Miller PJO, Madsen PT (2008) Shallow food for deep divers: dynamic foraging behavior of male sperm whales in a high latitude habitat. J Exp Mar Biol Ecol 354:119-131

Tyack PL (1983) Differential response of humpback whales,

Editorial responsibility: Brandon Southall,

Silver Spring, Maryland, USA
Megaptera novaeagliae, to playback of song or social sounds. Behav Ecol Sociobiol 13:49-55

Tyack PL (2008) Implications for marine mammals of largescale changes in the marine acoustic environment. J Mammal 89:549-558

Tyack PL, Zimmer WMX, Moretti D, Southall BL and others (2011) Beaked whales respond to simulated and actual navy sonar. PLOS ONE 6:e17009

Visser F, Miller P, Antunes R, Oudejans M and others (2014) The social context of individual foraging behaviour in long-finned pilot whales. Behaviour 151:1453-1477

Wartzok D, Popper AN, Gordon J, Merrill J (2003) Factors affecting the responses of marine mammals to acoustic disturbance. Mar Technol Soc J 37:6-15

Whitehead H (2006) Sperm whales in ocean ecosystems. In: Estes JA, DeMaster DP, Doak DF, Williams TM, Brownell RL (eds) Whales, whaling and ocean ecosystems. University of California Press, Berkeley, CA, p 324-334

Submitted: October 14, 2015; Accepted: July 18, 2016

Proofs received from author(s): September 16, 2016 